\title{
ANT-BASED EXTRACTION OF RULES IN SIMPLE DECISION SYSTEMS OVER ONTOLOGICAL GRAPHS
}

\author{
KRZYSZTOF PANCERZ ${ }^{a, b}$, ARKADIUSZ LEWICKI ${ }^{b}, \operatorname{RYSZARD~TADEUSIEWICZ~}^{c, *}$ \\ ${ }^{a}$ Department of Computer Science and Knowledge Engineering \\ University of Management and Administration, Akademicka 4, 22-400 Zamość, Poland \\ e-mail: kpancerz@wszia.edu.pl \\ ${ }^{b}$ Department of Applied Informatics \\ University of Information Technology and Management, Sucharskiego 2, 35-225 Rzeszów, Poland \\ e-mail: alewicki@wszia.edu.pl \\ ${ }^{c}$ Department of Automatic Control and Biomedical Engineering \\ AGH University of Science and Technology, Mickiewicza 30, 30-059 Kraków, Poland \\ e-mail: rtadeagh.edu.pl
}

\begin{abstract}
In the paper, the problem of extraction of complex decision rules in simple decision systems over ontological graphs is considered. The extracted rules are consistent with the dominance principle similar to that applied in the dominancebased rough set approach (DRSA). In our study, we propose to use a heuristic algorithm, utilizing the ant-based clustering approach, searching the semantic spaces of concepts presented by means of ontological graphs. Concepts included in the semantic spaces are values of attributes describing objects in simple decision systems.
\end{abstract}

Keywords: ant-based clustering, decision systems, DRSA, ontological graphs, rule extraction.

\section{Introduction}

Rough set theory delivers useful methods for knowledge discovery and data mining (cf. Pawlak, 1991). Starting points for various data mining algorithms, including rough set ones, are information (decision) tables consisting of vector descriptions of objects. In rough set theory, information (decision) tables are tabular representations of mathematical entities called information (decision) systems. In information (decision) tables, rows represent objects whereas columns correspond to attributes (features) of objects. Entries of the table (intersections of rows and columns) are values of attributes (corresponding to columns) describing objects (corresponding to rows). In classic approaches, values in information (decision) tables can be both symbolic and numeric. To compare objects by means of such attribute values, a lot of measures have been defined. First of all, there are various similarity measures of objects. A generalized definition of approximations of sets, based on similarity, was

* Corresponding author proposed, for example, by Slowinski and Vanderpooten (1996). Some approaches use also data semantics, e.g., the dominance-based rough set approach (Greco et al., 2001).

Intelligent systems play an important role in modern computer science (Tadeusiewicz, 2010b; 2011) and related fields. The recent research in the area of intelligent systems shows that, in many situations, data alone are not sufficient. There is a need to add some expert knowledge about relationships within data expressing the meaning of data. Such knowledge is included in ontologies. In the works of Pancerz (2012b; 2013b), ontologies were incorporated into information (decision) systems, i.e., attribute values were considered in the ontological (semantic) spaces. Similar approaches have been considered in the literature, e.g., DAG-decision systems (Midelfart and Komorowski, 2002), the dominance-based rough set approach (DRSA) (Greco et al., 2001), rough ontology (Ishizu et al., 2007), etc. In our approach, we replace, in a classic definition 
of information (decision) systems, simple sets of attribute values by ontological graphs which deliver us some new knowledge about meanings of attribute values. For this case, decision rules in decision systems can be seen from different perspectives, for example, taking into consideration synonymy, generality or some more sophisticated properties determining meanings of attribute values.

Formally, the ontology can be represented by means of graph structures. The graph representing the ontology is called the ontological graph. In such a graph, each node represents one concept from the ontology, whereas each edge represents a relation between two concepts. Relations are very important components in ontology modeling as they describe the relationships that can be established between concepts. There have been proposed two ways for creating information (decision) systems over ontological graphs. In the first approach, attribute values of a given information (decision) system are concepts from ontologies assigned to attributes. Such a system is said to be a simple information (decision) system over ontological graphs. In the second approach, attribute values of a given information (decision) system are local ontological subgraphs of ontological graphs assigned to attributes. Such a system is said to be a complex information (decision) system over ontological graphs. It means that the term "simple" should be understood as the word describing the property of the decision system, not the size of it. In simple decision systems over ontological graphs, attribute values are single concepts. The opposed system is "complex". In complex systems, attribute values are graph structures.

An important problem concerning decision systems is extracting the knowledge hidden in such systems. This knowledge can be expressed in the form of decision rules. The topic of rule definition and extraction in various decision systems has been widely considered in the literature. Therefore, Pancerz (2013a) defined decision rules and related notions in simple decision systems over ontological graphs analogously to those defined for classic decision systems in rough set theory.

Another look, this time based on the dominance-based rough set approach (Greco et al., 2001), at decision rules for simple decision systems over ontological graphs was presented by Pancerz (2012a), too. In that paper, elementary decision rules, similar to that defined in the DRSA, were considered. An elementary rule is said to be a rule with one condition descriptor. Moreover, the exhaustive algorithm for mining the most general elementary rules, in a given simple decision system over ontological graphs, with respect to their condition parts for fixed decision parts, was proposed. That algorithm was based on the depth-first search technique with pre-pruning.

The idea of incorporating the DRSA into decision systems over ontological graphs, proposed by Pancerz (2012a), is recalled in Section 3 In this paper, we continue the discussion on decision rules consistent with the dominance-based rough set approach. Our investigations are extended to the decision rules with complex condition parts, i.e., the rules having multi-descriptor left-hand sides. However, only a special case is taken into consideration, when descriptors appearing on the left-hand sides of rules are linked by the and logical connectives. The complex decision rules in simple decision systems over ontological graphs are considered in Section 4 In the case of complex rules, the rule extraction becomes a more complicated process than in classic rough set theory. The space of possible condition descriptors appearing in the rules is significantly greater. Descriptors can include not only attribute values (concepts) present in decision systems, but also values which are more general concepts than the ones mentioned, according to defined ontological graphs. Therefore, to solve the problem considered, we propose, in Section 5, to use some heuristic algorithm utilizing the ant-based clustering approach.

\section{Simple decision systems over ontological graphs}

Information (decision) systems were proposed by Pawlak (1991) as knowledge representation systems. An information (decision) system represents a set of objects described by attribute value vectors. Pancerz (2012b) proposed to consider attribute values describing objects in the ontological spaces, where ontologies are constructed on the basis of controlled vocabularies and the relationships of the concepts in the controlled vocabularies (cf. definitions given by Neches et al. (1991) and Köhler et al. (2006)). In that approach, we use formal representations of ontologies by means of graph structures. Such structures are called ontological graphs. For a given ontology $\mathcal{O}$, an ontological graph includes nodes representing concepts from $\mathcal{O}$ and edges representing relations between concepts from $\mathcal{O}$.

Definition 1. Let $\mathcal{O}$ be a given ontology. An ontological graph is a quadruple $O G=(\mathcal{C}, E, \mathcal{R}, \rho)$, where $\mathcal{C}$ is a nonempty, finite set of nodes representing concepts in the ontology $\mathcal{O}, E \subseteq \mathcal{C} \times \mathcal{C}$ is a finite set of edges representing relations between concepts from $\mathcal{C}, \mathcal{R}$ is a family of semantic descriptions (in natural language) of types of relations (represented by edges) between concepts, and $\rho: E \rightarrow \mathcal{R}$ is a function assigning a semantic description of the relation to each edge.

Semantic relations describe the relationships that can be established between concepts. In the literature, a variety of taxonomies of different types of semantic relations has been proposed (Brachman, 1983; Chaffin 
and Herrmann, 1988; Milstead, 2001; Storey, 1993; Winston et al., 1987). In our approach, we will use the taxonomy of types of semantic relations modeled on the project called Wikisaurus (Wikisaurus, 2013) aiming at creating a thesaurus of semantically related terms.

There are four main types of semantic relations distinguished in the project: synonymy, antonymy, hyponymy/hyperonymy, meronymy/holonymy. Synonymy concerns concepts with a meaning that is the same as, or very similar to, other concepts. Antonymy concerns concepts which have the opposite meaning to others. Both of these relations are nonhierarchical. Hyponymy/hyperonymy determines narrower/broader meaning. Hyponymy concerns more specific concepts than others. Hyperonymy concerns more general concepts than others. Meronymy and holonymy define part/whole relations. Meronymy concerns concepts that denote parts of the wholes that are denoted by other concepts. Holonymy concerns concepts that denote wholes whose parts are denoted by other concepts.

Further, we will be interested only in the hyperonymy. This relation will be marked with $R_{\triangleright}$ and $\left(v, v^{\prime}\right) \in R_{\triangleright}$ is read " $v$ is a hyperonym of $v^{\prime \prime}$. This label will be used, for simplicity, instead of a semantic description (in natural language) of hyperonymy assigned to edges in ontological graphs. In drawing ontological graphs, for readability, we will omit reflexivity of hyperonymy. However, a given concept is a hyperonym of itself.

In this paper, we will use the definition of a simple decision system over ontological graphs given by Pancerz (2012b).

Definition 2. A simple decision system $S D S^{O G}$ over ontological graphs is the septuple

$$
S D S^{O G}=\left(U, C, D,\left\{O G_{a}\right\}_{a \in C}, V_{d}, f_{c}, f_{d}\right),
$$

such that

- $U$ is a nonempty, finite set of objects,

- $C$ is a nonempty, finite set of condition attributes,

- $D$ is a nonempty, finite set of decision attributes,

- $\left\{O G_{a}\right\}_{a \in C}$ is a family of ontological graphs associated with condition attributes from $C$,

- $V_{d}=\bigcup_{a \in D} V_{a}$, where $V_{a}$ is a set of values of the decision attribute $a \in D$,

- $f_{c}: C \times U \rightarrow \mathcal{C}$, where $\mathcal{C}=\bigcup_{a \in C} \mathcal{C}_{a}$, is an information function such that $f_{c}(a, u) \in \mathcal{C}_{a}$ for each $a \in C$ and $u \in U$, where $\mathcal{C}_{a}$ is a set of concepts from the graph $O G_{a}$,

- $f_{d}: D \times U \rightarrow V_{d}$ is a decision function such that $f_{d}(a, u) \in V_{d}$ for each $a \in D$ and $u \in U$.
Remark 1. It is not necessary for an information function to be a total function, i.e., $f_{c}: C \times U \rightarrow \mathcal{C}^{*} \subseteq \mathcal{C}$.

The character of sets of attribute values differentiates simple information (decision) systems over ontological graphs from information (decision) systems proposed by Pawlak (1991). Now, attribute values are not singular (individual) values, but they are placed in the graph structures expressing relationships between these values.

Pancerz (2014) developed complex information systems over ontological graphs in which attribute values are local ontological graphs of ontologies assigned to attributes.

Example 1. (Decision system) Let $S D S^{O G}=$ $\left(U, C, D,\left\{O G_{a}\right\}_{a \in C}, V_{d}, f_{c}, f_{d}\right)$ be a simple decision system, represented by a decision table (see Table 1), over ontological graphs shown in Figs. 1 and 2 In this system,

$$
U=\left\{u_{1}, u_{2}, \ldots, u_{15}\right\}
$$

is a set of fifteen objects described with respect to the development level. $C=\{$ Sector, Region $\}$ is a set of condition attributes describing an economy sector and a continental region. $D=\{$ Level $\}$ is a set of decision attributes, $D$ consists of one attribute evaluating the development level. $O G_{\text {Sector }}=\left(\mathcal{C}_{S}, E_{S}, \mathcal{R}, \rho_{S}\right)$ is an ontological graph associated with the attribute Sector (see Fig. 1). $O G_{\text {Region }}=\left(\mathcal{C}_{R}, E_{R}, \mathcal{R}, \rho_{R}\right)$ is an ontological graph associated with the attribute Region (see Fig. 2). As mentioned earlier, only hyperonymy is taken into consideration, i.e., $\mathcal{R}=\left\{R_{\triangleright}\right\} . \quad V_{d}=$ $\{$ Low, Medium, High $\}$ is a set of decision values. $f_{c}$ is an information function and $f_{d}$ is a decision function, both defined in the tabular form in Table 1.

Obviously, ontological graphs used in this example are simplified in comparison to ontological graphs expressing real-world relations between concepts.

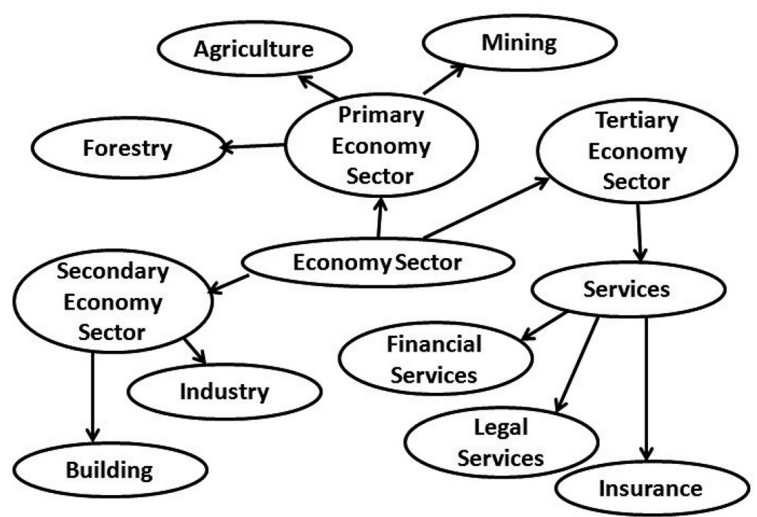

Fig. 1. Ontological graph $O G_{\text {Sector }}$ associated with the attribute Sector. 
Table 1. Simple decision system over ontological graphs.

\begin{tabular}{|c||c|c||c|}
\hline$U / C \cup D$ & Sector & Region & Level \\
\hline \hline$u_{1}$ & Forestry & Northern America & High \\
\hline$u_{2}$ & Forestry & Caribbean & Medium \\
\hline$u_{3}$ & Forestry & Latin America & Medium \\
\hline$u_{4}$ & Forestry & Middle East & Low \\
\hline$u_{5}$ & Mining & Middle East & High \\
\hline$u_{6}$ & Financial Services & Northern America & High \\
\hline$u_{7}$ & Legal Services & Northern America & High \\
\hline$u_{8}$ & Insurance & Northern America & High \\
\hline$u_{9}$ & Financial Services & Latin America & Medium \\
\hline$u_{10}$ & Legal Services & Latin America & Medium \\
\hline$u_{11}$ & Insurance & Latin America & Medium \\
\hline$u_{12}$ & Industry & Far East & High \\
\hline$u_{13}$ & Industry & Asia Pacific & Medium \\
\hline$u_{14}$ & Industry & Middle East & Medium \\
\hline$u_{15}$ & Mining & Far East & Low \\
\hline
\end{tabular}

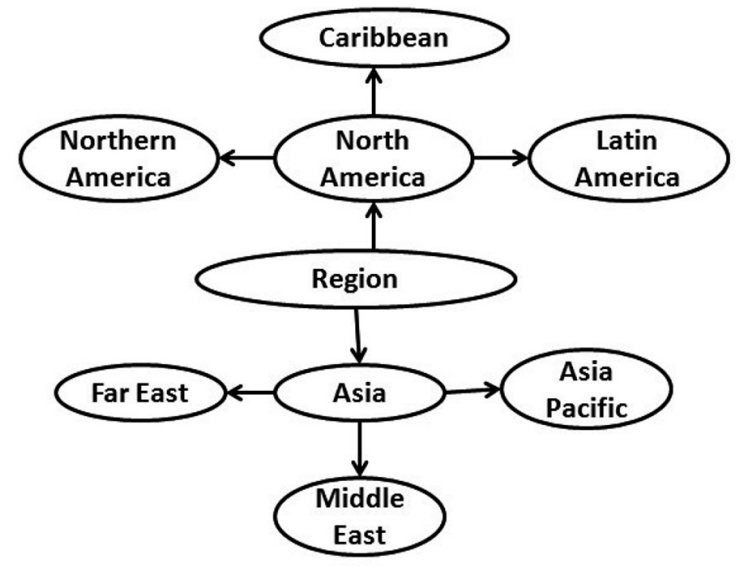

Fig. 2. Ontological graph $O G_{\text {Region }}$ associated with the attribute Region.

Let $O G=(\mathcal{C}, E, \mathcal{R}, \rho)$ be an ontological graph. In further definitions, we will use the following notation: $\left[c_{i}, c_{j}\right]$ is a simple path in $O G$ between $c_{i}, c_{j} \in \mathcal{C}$, $\mathcal{E}\left(\left[c_{i}, c_{j}\right]\right)$ is a set of all edges from $E$ belonging to the simple path $\left[c_{i}, c_{j}\right]$, and $\mathcal{P}(O G)$ is a set of all simple paths in $O G$. In the literature, there are different definitions for a simple path in the graph. In this paper, we follow the definition in which a path is simple if no node or edge is repeated, with the possible exception that the first node is the same as the last. Therefore, the path $\left[c_{i}, c_{j}\right]$, where $c_{i}, c_{j} \in \mathcal{C}$ and $c_{i}=c_{j}$ can also be a simple path in $O G$.

Definition 3. Given an ontological graph $O G_{a}=$ $\left(\mathcal{C}_{a}, E_{a}, \mathcal{R}, \rho_{a}\right)$ associated with the attribute $a$ in a simple decision system, where $\mathcal{R}=\left\{R_{\triangleright}\right\}$ and $v \in \mathcal{C}_{a}$ a hyperonymous meaning relation $\operatorname{HprMR}_{a}^{v}$ is a set of all pairs $\left(c_{1}, c_{2}\right) \in \mathcal{C}_{a} \times \mathcal{C}_{a}$ satisfying the following conditions:

$$
\underset{\left[v, c_{1}\right] \in \mathcal{P}\left(O G_{a}\right)}{\exists}\left(\underset{e \in \mathcal{E}\left(\left[v, c_{1}\right]\right)}{\forall} \rho(e) \in\left\{R_{\triangleright}\right\}\right)
$$

and

$$
\underset{\left[v, c_{2}\right] \in \underset{\mathcal{P}}{\exists}\left(O G_{a}\right)}{\exists}\left(\underset{e \in \mathcal{E}\left(\left[v, c_{2}\right]\right)}{\forall} \rho(e) \in\left\{R_{\triangleright}\right\}\right) .
$$

If two concepts are in the hyperonymous meaning relation, then in a more general meaning, they can be treated as the same concept, for example, car and bus are, in a more general meaning, vehicle.

\section{Dominance-based rough set approach in simple decision systems over ontological graphs}

Pancerz (2012a) proposed to consider simple decision systems over ontological graphs in terms of the dominance-based rough set approach (Greco et al., 2001). It seems to be natural because hyperonymy can be considered in terms of dominance relations.

Let an ontological graph $O G_{a}=\left(\mathcal{C}_{a}, E_{a}, \mathcal{R}, \rho_{a}\right)$ be associated with the attribute $a$ in a simple decision system and $c_{1}, c_{2} \in \mathcal{C}_{a}$. It is said that $c_{1}$ dominates $c_{2}$, written as $D^{\geq}\left(c_{1}, c_{2}\right)$, if $c_{2}$ is a hyperonym of $c_{1}$ (or, in other words, $c_{1}$ is a hyponym of $c_{2}$ ).

Let an ontological graph $O G_{a}=\left(\mathcal{C}_{a}, E_{a}, \mathcal{R}, \rho_{a}\right)$ be associated with the attribute $a$ in a simple decision system and $c_{1}, c_{2} \in \mathcal{C}_{a}$. It is said that $c_{1}$ is dominated by $c_{2}$, written as $D^{\leq}\left(c_{1}, c_{2}\right)$, if $c_{1}$ is a hyperonym of $c_{2}$ (or, in other words, $c_{2}$ is a hyponym of $c_{1}$ ).

Remark 2. According to the definitions given earlier, we will further denote the hyponymy relation by $D^{\geq}\left(c_{1}, c_{2}\right)$ and the hyperonymy relation by $D \leq\left(c_{1}, c_{2}\right)$. 
Example 2. (Domination) Consider the ontological graph $O G_{\text {Region }}$ given in Example1. For instance, Middle East dominates Asia, i.e., $\quad D^{\geq}$(MiddleEast, Asia), whereas Asia is dominated by Middle East, i.e., $D \leq($ Asia, Middle East $)$.

In the dominance-based rough set approach (Greco et al., 2001), an outranking relation $S_{q}$ (Roy, 1985) corresponding to a criterion $q$ is used. In this case, $S_{q}(x, y)$ means that " $x$ is at least as good as $y$ with respect to the criterion $q$ ". In our approach, covering hyperonymy, the meaning will be quite similar, i.e., " $x$ is at least $y$ with respect to a given ontological graph $O G$ ".

Definition 4. Consider a simple decision system

$$
S D S^{O G}=\left(U, C, D,\left\{O G_{a}\right\}_{a \in C}, V_{d}, f_{c}, f_{d}\right)
$$

over ontological graphs, $a \in C$ and $u \in U$. An $a$-dominating set with respect to $u$ is the set

$$
D_{f_{c}(a, u)}^{+}=\left\{u^{\prime} \in U: D^{\geq}\left(f_{c}(a, u), f_{c}\left(a, u^{\prime}\right)\right)\right\} .
$$

An $a$-dominated set with respect to $u$ is a set

$$
D_{f_{c}(a, u)}^{-}=\left\{u^{\prime} \in U: D^{\leq}\left(f_{c}(a, u), f_{c}\left(a, u^{\prime}\right)\right)\right\} .
$$

Remark 3. Consider a simple decision system

$$
S D S^{O G}=\left(U, C, D,\left\{O G_{a}\right\}_{a \in C}, V_{d}, f_{c}, f_{d}\right)
$$

over ontological graphs be given, $a \in C$ and $v \in \mathcal{C}_{a}$ of $O G_{a}$. We use the following notation:

- by $D_{a}^{+v}$ we denote the set

$$
\left\{u \in U: D^{\geq}\left(f_{c}(a, u), v\right)\right\},
$$

i.e., the set of all objects $u$ in $U$ for which $f_{c}(a, u)$ dominates $v$,

- by $D_{a}^{-v}$ we denote the set

$$
\left\{u \in U: D^{\leq}\left(f_{c}(a, u), v\right)\right\},
$$

i.e., the set of all objects $u$ in $U$ for which $f_{c}(a, u)$ is dominated by $v$.

The $a$-dominating set with respect to $u$ is a set of all objects from $U$ having concepts assigned to them by the attribute $a$ whose hyperonym is the concept assigned to $u$ by the attribute $a$ according to an ontological graph $O G_{a}$. Analogously, the $a$-dominated set with respect to $u$ is a set of all objects from $U$ having concepts assigned to them by the attribute $a$ which are hyperonyms of the concept assigned to $u$ by the attribute $a$ according to an ontological graph $O G_{a}$.

\section{Decision rules consistent with the dominance principle}

In this section, we use definitions related to the dominance-based rough set approach, given, among other things, by Greco et al. (2001), to provide notions for decision rules in a simple decision system over ontological graphs.

Let $S D S^{O G}=\left(U, C, D,\left\{O G_{a}\right\}_{a \in C}, V_{d}, f_{c}, f_{d}\right)$ be a simple decision system over ontological graphs, $a_{d} \in D$ and $\mathbf{C l}_{a_{d}}=\left\{C l_{t}: t \in T\right\}$, where $T=\{1, \ldots, n\}$, be a set of classes of $U$ determined by $a_{d}$, such that $u \in U$ belongs to one and only one class $C l_{t} \in \mathbf{C l}_{a_{d}}$. Moreover, suppose that we can define a complete preorder, i.e., a strongly complete and transitive binary relation $S_{a_{d}}$, for a decision attribute $a_{d} \in D$ in $S D S^{O G}$. For $r, s \in T$, $r>s$ means that each element of $C l_{r}$ is preferred (strictly or weakly) to each element of $C l_{s}$. For $u, v \in U$, $(u, v) \in S_{a_{d}}$ means " $u$ is at least as good as $v$ ".

For a family $\mathbf{C l}_{a_{d}}$ of classes, we define an upward union of classes:

$$
C l_{t}^{\geq}=\bigcup_{s \geq t} C l_{s}
$$

where $C l_{t}, C l_{s} \in \mathbf{C l}_{a_{d}}$.

Let $S D S^{O G}=\left(U, C, D,\left\{O G_{a}\right\}_{a \in C}, V_{d}, f_{c}, f_{d}\right)$ be a simple decision system over ontological graphs, $a \in C$ and $C l_{t}^{\geq}$be an upward union of classes determined by a given decision attribute from $D$. We define

- the $a$-lower approximation of $C l_{t}^{\geq}$:

$$
\underline{a}\left(C l_{t}^{\geq}\right)=\left\{u \in U: D_{a}^{+}(u) \subseteq C l_{t}^{\geq}\right\},
$$

- the $a$-upper approximation of $C l_{t}^{\geq}$:

$$
\bar{a}\left(C l_{t}^{\geq}\right)=\bigcup_{u \in C l_{t}^{\geq}} D_{a}^{+}(u),
$$

Let $S D S^{O G}=\left(U, C, D,\left\{O G_{a}\right\}_{a \in C}, V_{d}, f_{c}, f_{d}\right)$ be a simple decision system over ontological graphs. Let $\mathcal{C}=$ $\bigcup_{a \in C} \mathcal{C}_{a}$, where $\mathcal{C}_{a}$ is a set of concepts from the graph $O G_{a}$ associated with a given $a \in C$. For the decision system $S D S^{O G}$, we define

- condition descriptors, which are expressions $(a, v) \geq$ over $C$ and $\mathcal{C}$, where $a \in C$ and $v \in \mathcal{C}$, read as " $a$ is at least $v$ " according to $O G_{a}$,

- decision descriptors, which are expressions $(a, v) \geq$ over $D$ and $V_{d}$, where $a \in D$ and $v \in V_{d}$, read as " $a$ is at least $v$ " according to a complete preorder defined for $a$.

In a given simple decision system

$$
S D S^{O G}=\left(U, C, D,\left\{O G_{a}\right\}_{a \in C}, V_{d}, f_{c}, f_{d}\right)
$$


over ontological graphs, we will consider a $D_{>}$-decision rule in the form

$$
\begin{array}{r}
\left(a_{c_{1}}, v_{c_{1}}\right)^{\geq} \wedge\left(a_{c_{2}}, v_{c_{2}}\right)^{\geq} \wedge \cdots \wedge\left(a_{c_{k}}, v_{c_{k}}\right)^{\geq} \\
\Rightarrow\left(a_{d}, v_{d}\right)^{\geq},
\end{array}
$$

where $a_{c_{1}}, a_{c_{2}}, \ldots, a_{c_{k}} \in C, v_{c_{1}} \in \mathcal{C}_{a_{c_{1}}}$ of $O G_{a_{c_{1}}}, v_{c_{2}} \in$ $\mathcal{C}_{a_{c_{2}}}$ of $O G_{a_{c_{2}}}, \ldots, v_{c_{k}} \in \mathcal{C}_{a_{c_{k}}}$ of $O G_{a_{c_{k}}}, a_{d} \in D$, $v_{d} \in V_{d}$ and $\phi_{1} \wedge \phi_{2} \wedge \cdots \wedge \phi_{k} \Rightarrow \psi$ is read: "if $\phi_{1}$ and $\phi_{2}$ and ... and $\phi_{k}$, then $\psi "$.

The above decision rule can be read in the following way: "if $a_{c_{1}}$ is at least $v_{c_{1}}$ and $a_{c_{2}}$ is at least $v_{c_{2}}$ and $\ldots$ and $a_{c_{k}}$ is at least $v_{c_{k}}$, then $a_{d}$ is at least $v_{d}$ ". This rule is true (valid, certain) in $S D S^{O G}$ if and only if

$$
D_{a_{c_{1}}}^{+v_{c_{1}}} \cap D_{a_{c_{2}}}^{+v_{c_{2}}} \cap \cdots \cap D_{a_{c_{k}}}^{+v_{c_{k}}} \subseteq C l_{v_{d}}^{\geq}
$$

and

$$
D_{a_{c_{1}}}^{+v_{c_{1}}} \cap D_{a_{c_{2}}}^{+v_{c_{2}}} \cap \cdots \cap D_{a_{c_{k}}}^{+v_{c_{k}}} \neq \emptyset,
$$

where $C l_{v_{d}}$ denotes the class of objects $u \in U$ such that $f_{d}\left(a_{d}, u\right)=v_{d}$.

Remark 4. If, in the above rule, $k=1$, then the rule is called a $D_{\geq}$-elementary decision rule, i.e., it has the form

$$
\left(a_{c}, v_{c}\right)^{\geq} \Rightarrow\left(a_{d}, v_{d}\right)^{\geq},
$$

where $a_{c} \in C, v_{c} \in \mathcal{C}_{a_{c}}$ of $O G_{a_{c}}, a_{d} \in D, v_{d} \in V_{d}$.

Investigations into $D_{\geq}$-elementary decision rules in simple decision systems over ontological graphs were carried out by Pancerz (2012a).

From rough set theory, we know that lower approximations generate rules true in decision systems. In our case, each nonempty $B$-lower approximation of $C l_{t}^{\geq}$ generates $D_{\geq}$-elementary decision rules true in a given decision system, where $B=\left\{a_{c_{1}}, a_{c_{2}}, \ldots, a_{c_{k}}\right\}$.

\section{Generation of decision rules consistent with the dominance principle}

Pancerz (2013a) extended notions related to decision rules in classic decision systems, given by Pawlak (1991), to analogous notions for simple decision systems over ontological graphs. Another look, this time based on the dominance-based rough set approach (DRSA) (Greco et al., 2001), at decision rules for simple decision systems over ontological graphs was presented also by Pancerz (2012a). However, in the second case, we considered only the most general elementary rules true in a given simple decision system over ontological graphs.

Let $S D S^{O G}=\left(U, C, D,\left\{O G_{a}\right\}_{a \in C}, V_{d}, f_{c}, f_{d}\right)$ be a simple decision system over ontological graphs. An elementary rule $\left(a_{c}, v_{c}\right) \geq \Rightarrow\left(a_{d}, v_{d}\right) \geq$, where $a_{c} \in C$, $v_{c} \in \mathcal{C}_{a_{c}}$ of $O G_{a_{c}}, a_{d} \in D, v_{d} \in V_{d}$, is said to be the most general rule with respect to its condition part for a fixed decision part $\left(a_{d}, v_{d}\right) \geq$ if and only if the rule $\left(a_{c}, v_{c}\right)^{\geq} \Rightarrow\left(a_{d}, v_{d}\right)^{\geq}$is true in $S D S^{O G}$, but the rule $\left(a_{c}, v_{c}^{\prime}\right)^{\geq} \Rightarrow\left(a_{d}, v_{d}\right) \geq$, where $v_{c}^{\prime}$ is a hyperonym of $v_{c}$ according to $O G_{a_{c}}$, is not true in $S D S^{O G}$.

In this paper, we consider a more general case, i.e., decision rules have the form mentioned in Section 4 Such a situation complicates the problem of extraction of rules, consistent with the dominance principle, from simple decision systems over ontological graphs. In the remaining part of this section, we propose some heuristic algorithm (see Algorithm 1) using the ant-based clustering process for extraction of decision rules true in a given simple decision system over ontological graphs. At the beginning, we start with some auxiliary notions used in the ant-based algorithm.

$$
\begin{aligned}
& \text { Let } \\
& \begin{aligned}
r:\left(a_{c_{1}}, v_{c_{1}}\right) \geq \wedge & \left(a_{c_{2}}, v_{c_{2}}\right) \geq \wedge \cdots \wedge\left(a_{c_{k}}, v_{c_{k}}\right) \geq \\
& \Rightarrow\left(a_{d}, v_{d}\right) \geq
\end{aligned}
\end{aligned}
$$

be a $D_{>}$-decision rule and

$$
\begin{gathered}
U_{r}^{\not E}=\left(D_{a_{c_{1}}}^{+v_{c_{1}}} \cap D_{a_{c_{2}}}^{+v_{c_{2}}} \cap \cdots \cap D_{a_{c_{k}}}^{+v_{c_{k}}}\right)-C l_{v_{d}}^{\geq}, \\
U_{r}^{c}=D_{a_{c_{1}}}^{+v_{c_{1}}} \cap D_{a_{c_{2}}}^{+v_{c_{2}}} \cap \cdots \cap D_{a_{c_{k}}}^{+v_{c_{k}}} .
\end{gathered}
$$

For the rule $r$, we define its accuracy $a c c(r)$ as

$$
\operatorname{acc}(r)=\frac{\operatorname{card}\left(U_{r}^{c}\right)-\operatorname{card}\left(U_{r}^{\not \neq}\right)}{\operatorname{card}\left(U_{r}^{c}\right)},
$$

where $\operatorname{card}(X)$ denotes the cardinality of $X$

It is easy to see that if $r$ is true in a given simple decision system over ontological graphs, then $U_{r}^{\not \nvdash}=\emptyset$ and $\operatorname{acc}(r)=1$.

Let $S D S^{O G}=\left(U, C, D,\left\{O G_{a}\right\}_{a \in C}, V_{d}, f_{c}, f_{d}\right)$ be a simple decision system over ontological graphs, where $D=\left\{a_{d}\right\}$. For each ontological graph $O G_{a}=$ $\left(\mathcal{C}_{a}, E_{a}, \mathcal{R}, \rho_{a}\right)$, where $a \in C$, for each concept (attribute value) $v \in \mathcal{C}_{a}$, we define the goodness $\delta_{v}$ of $v$ for a fixed $v_{d} \in V_{d}$ as

$$
\delta_{v}=\frac{\operatorname{card}\left(D_{a}^{+v}\right)-\operatorname{card}\left(D_{a}^{+v}-C l_{\bar{v}_{d}}^{\geq}\right)}{\operatorname{card}\left(D_{a}^{+v}\right)} .
$$

It is easy to see that the goodness of a given concept $v$ is equal to 1 with respect to a fixed $v_{d} \in V_{d}$ if and only if a $D_{\geq}$-elementary decision rule

$$
(a, v)^{\geq} \Rightarrow\left(a_{d}, v_{d}\right)^{\geq}
$$

is true in $S D S^{O G}$.

Example 3. (Elementary rules) Let us consider the simple decision system over ontological graphs $S D S^{O G}$ given in Example 1] For a fixed decision part in the form $C l \stackrel{\text { Medium }}{\geq}$, the concepts from ontological graphs $O G_{\text {Sector }}$ and $O G_{\text {Region }}$ with the goodness equal to 1 are marked in gray in Figs. 3 and 4 respectively.

According to Figs. 3 and 4 , we obtain the following $D_{\geq}$-elementary decision rules true in $S D S^{O G}$ : 


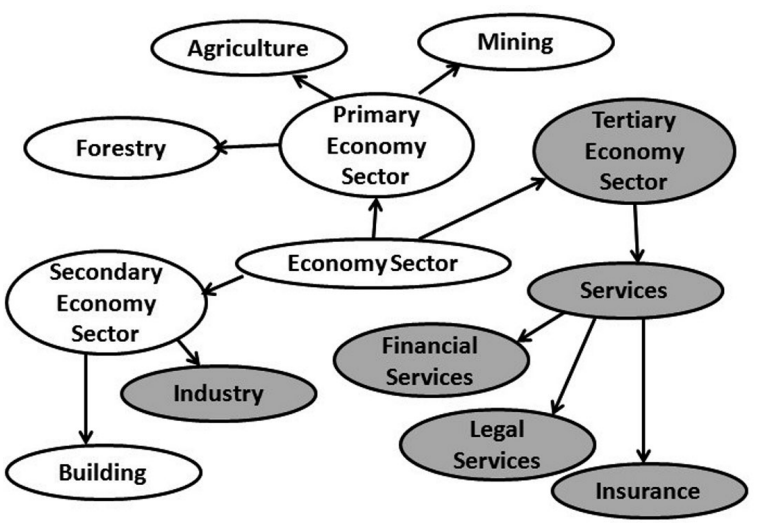

Fig. 3. Concepts with the goodness equal to 1 (marked in gray) in ontological graph $O G_{\text {Sector }}$.

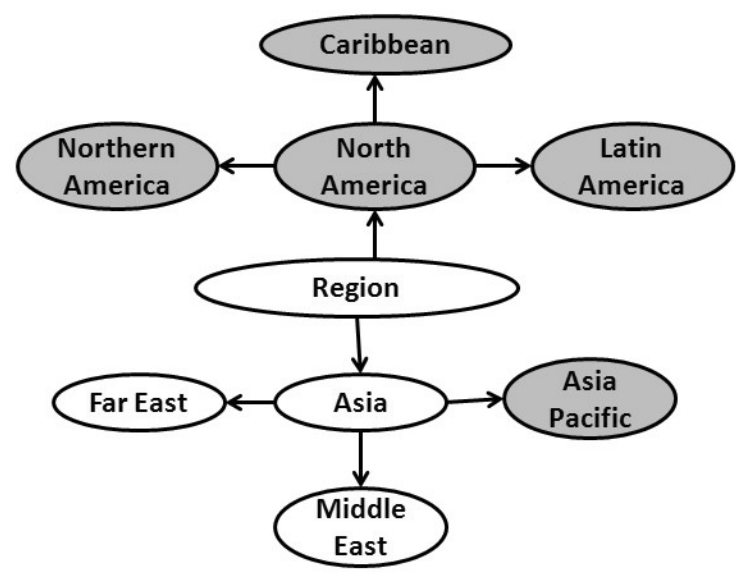

Fig. 4. Concepts with the goodness equal to 1 (marked in gray) in ontological graph $O G_{\text {Region }}$.

- (Sector, Tertiary Economy Sector $) \geq$ (Level, Medium $)^{\geq}$,

- $(\text { Sector }, \text { Services })^{\geq} \Rightarrow(\text { Level, Medium })^{\geq}$,

- $(\text { Sector, Financial Services })^{\geq} \Rightarrow$ (Level, Medium $)^{\geq}$,

- $(\text { Sector }, \text { Legal Services })^{\geq} \Rightarrow$ (Level, Medium $)^{\geq}$,

- $(\text { Sector }, \text { Insurance })^{\geq} \Rightarrow(\text { Level }, \text { Medium })^{\geq}$,

- $($ Sector, Industry $) \geq(\text { Level, Medium })^{\geq}$,

- $(\text { Region, North America })^{\geq} \Rightarrow$ (Level, Medium $)^{\geq}$,

- $($ Region, Northern America $) \geq \Rightarrow$ (Level, Medium $)^{\geq}$,

- $(\text { Region, Caribbean })^{\geq} \Rightarrow(\text { Level }, \text { Medium })^{\geq}$,
- $(\text { Region, Latin America })^{\geq} \Rightarrow$ (Level, Medium $)^{\geq}$,

- $(\text { Region, Asia Pacific })^{\geq} \Rightarrow$ (Level, Medium $) \geq$.

It is worth noting that we can select, from the above $D_{\geq}$-elementary decision rules, the most general ones:

- (Sector, Tertiary Economy Sector $) \geq \Rightarrow$ (Level, Medium $)^{\geq}$,

- $($ Sector, Industry $) \geq \Rightarrow(\text { Level, Medium })^{\geq}$,

- $(\text { Region, North America })^{\geq} \Rightarrow$ $(\text { Level, Medium })^{\geq}$,

- $(\text { Region, Asia Pacific })^{\geq} \Rightarrow$ (Level, Medium $)^{\geq}$.

Let $S D S^{O G}=\left(U, C, D,\left\{O G_{a}\right\}_{a \in C}, V_{d}, f_{c}, f_{d}\right)$ be a simple decision system over ontological graphs. Analogously to Pancerz (2012a), we are interested in mining decision rules, in a given simple decision system over ontological graphs, with respect to their condition parts for fixed decision parts $\left(a_{d}, v_{d}\right) \geq$. In the formal description of Algorithm 1] we can distinguish two main parts. In the first one, goodness for all concepts occurring in ontological graphs associated with condition attributes in $S D S^{O G}$ is calculated. Next, all concepts with the goodness equal to 1 are extracted from the graph because they generate elementary decision rules true in $S D S^{O G}$. These elementary rules are recorded. The remaining concepts participate in creating multi-condition descriptor decision rules in the second part of the algorithm. This part has a heuristic character utilizing the ant-based clustering process. The algorithm is mainly based on those proposed earlier by Deneubourg et al. (1991), Lumer and Faieta (1994) as well as Handl et al. (2006). Formulas for picking and dropping decisions are proposed to adjust the process to our specific problem. The aim of the ant-based clustering process is to join together descriptors including concepts from ontological graphs to build decision rules with complex condition parts, i.e., consisting of descriptors linked by the and logical connectives, but possibly with the smallest numbers of descriptors. Such rules are called minimal. The problem of extraction of minimal rules has been earlier considered in the literature (e.g., Fernández et al., 2001; Skowron and Rauszer, 1992).

The algorithm has the polynomial complexity. Let $\operatorname{Descr}(p)$ denote the set of descriptors occupying a given place $p$ because, in our approach, a given place $p$ can be occupied by more than one object, i.e., some heaps can be created. Picking and dropping decisions for 


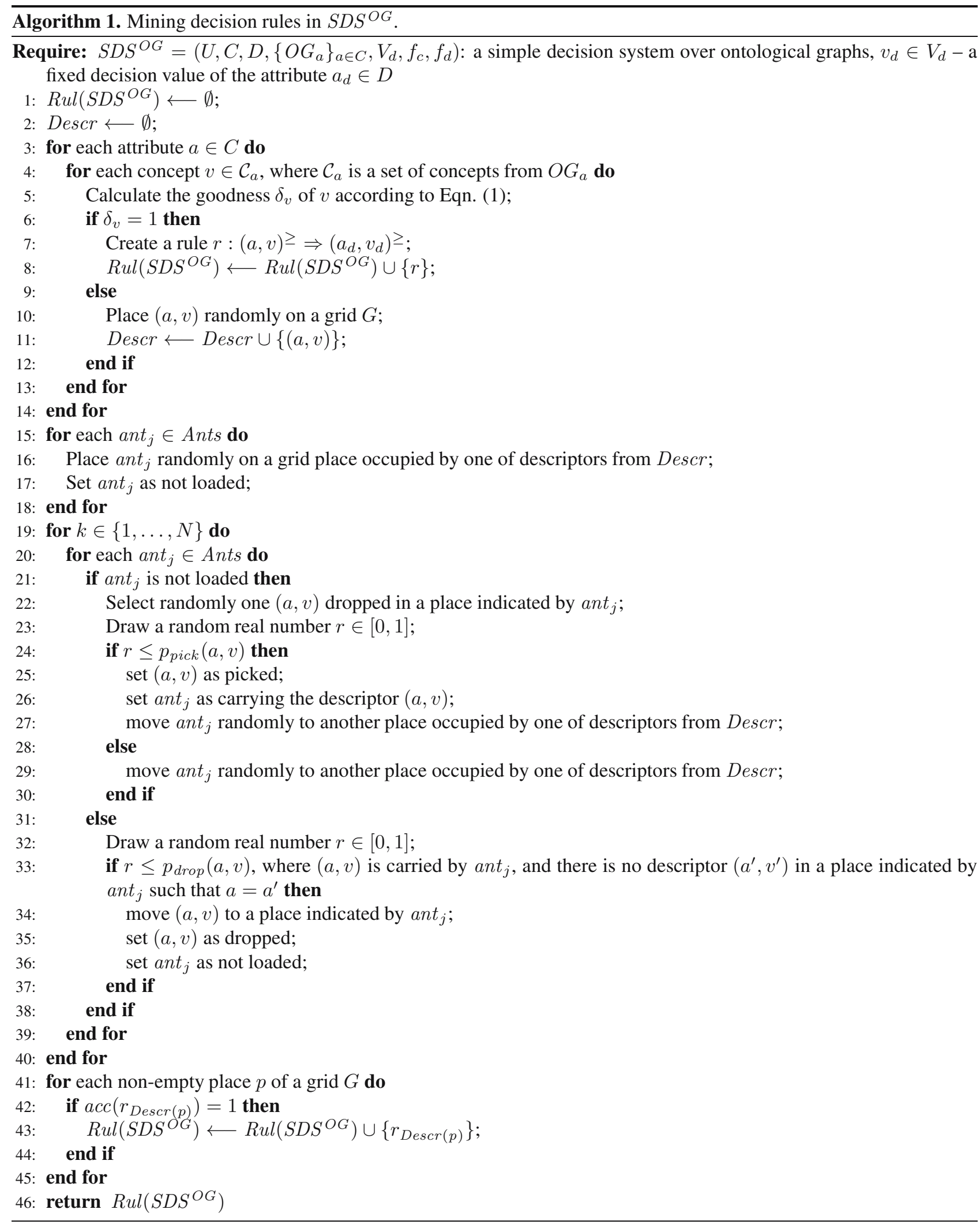


the descriptor $(a, v)$ can be formally expressed by the following formulas:

$$
p_{\text {pick }}(a, v)= \begin{cases}0 & \text { if } a c c_{r}=1, \\ 1-a c c_{r^{\prime}} & \text { otherwise }\end{cases}
$$

and

$$
p_{\text {drop }}(a, v)= \begin{cases}0 & \text { if } a c c_{r}=1, \\ a c c_{r^{\prime}} & \text { otherwise }\end{cases}
$$

where

- $r$ is a rule built on descriptors from $\operatorname{Descr}(p)$, i.e.,

$$
\begin{aligned}
r:\left(a_{c_{1}}, v_{c_{1}}\right) \geq & \wedge\left(a_{c_{2}}, v_{c_{2}}\right) \geq \wedge \cdots \wedge\left(a_{c_{k}}, v_{c_{k}}\right) \geq \\
& \Rightarrow\left(a_{d}, v_{d}\right) \geq
\end{aligned}
$$

- $r^{\prime}$ is a rule built on descriptors from $\operatorname{Descr}(p)$ and $(a, v)$, i.e.,

$$
\begin{aligned}
r^{\prime}:\left(a_{c_{1}}, v_{c_{1}}\right) & \geq \wedge\left(a_{c_{2}}, v_{c_{2}}\right) \geq \wedge \cdots \wedge\left(a_{c_{k}}, v_{c_{k}}\right) \geq \\
& \wedge(a, v)^{\geq} \Rightarrow\left(a_{d}, v_{d}\right) \geq
\end{aligned}
$$

- $\operatorname{Descr}(p)=\left\{\left(a_{c_{1}}, v_{c_{1}}\right),\left(a_{c_{2}}, v_{c_{2}}\right), \ldots,\left(a_{c_{k}}, v_{c_{k}}\right)\right\}$.

It is easy to see that, if a rule built on descriptors occupying a given place $p$ has the accuracy equal to 1 , then another descriptor is neither picked up nor dropped at this place. This enables us to obtain true decision rules with a minimal number of descriptors on the left-hand sides of the rules.

Remark 5. In Algorithm $1 r_{\operatorname{Descr}(p)}$ denotes a rule built on descriptors from $\operatorname{Descr}(p)$, i.e., if $\operatorname{Descr}(p)=$ $\left\{\left(a_{c_{1}}, v_{c_{1}}\right),\left(a_{c_{2}}, v_{c_{2}}\right), \ldots,\left(a_{c_{k}}, v_{c_{k}}\right)\right\}$, then

$$
\begin{gathered}
r_{\operatorname{Descr}(p)}:\left(a_{c_{1}}, v_{c_{1}}\right) \geq \wedge\left(a_{c_{2}}, v_{c_{2}}\right) \geq \wedge \ldots \\
\wedge\left(a_{c_{k}}, v_{c_{k}}\right)^{\geq} \Rightarrow\left(a_{d}, v_{d}\right)^{\geq} .
\end{gathered}
$$

Example 4. (Rules) Let us consider the simple decision system over ontological graphs $S D S^{O G}$ given in Example 1 and concepts with the goodness equal to 1 determined in Example 3. For mining multi-descriptor decision rules true in $S D S^{O G}$, we use the ant clustering process with the initial set of descriptors (the remaining descriptors, after removing those with the goodness equal to 1 , from ontological graphs $O G_{\text {Sector }}$ and $O G_{\text {Region }}$, i.e., those marked in gray in Figs. 3 and 4 respectively). After execution of the second part of Algorithm 11, we obtain, for example, the following $D_{\geq}$-decision rule true in $S D S^{O G}$, for fixed (Level, High) $\geq$ :

$$
\begin{gathered}
(\text { Sector }, \text { Mining })^{\geq \wedge}(\text { Region }, \text { Middle East }) \\
\Rightarrow(\text { Level }, \text { High })^{\geq} .
\end{gathered}
$$

\section{Conclusions and further work}

In the paper, we have proposed a heuristic algorithm based on the ant clustering for mining decision rules consistent with the dominance-based rough set approach in simple decision systems over ontological graphs. The algorithm consists of two stages. At the first stage, the algorithm is, in fact, deterministic and it extracts all $D_{\geq}$-elementary decision rules. At the second stage, the algorithm is properly heuristic and it extracts the $D_{\geq}$-decision rules, this time, with multiple descriptors on the left-hand sides of the rules. One can see in the paper that the presented approach refers to computations proposed years ago by Zadeh (1996) and called "computing with words". In further work, we will examine more complicated picking and dropping decision formulas enabling us to obtain decision rules, as general as possible, and we will consider another heuristics (e.g., genetic algorithms) for mining decision rules in simple decision systems over ontological graphs, and compare the quality of decision rules on real-life data.

\section{Acknowledgment}

This paper has been partially supported by the grant no. N N519 654540 from the National Science Centre in Poland.

\section{References}

Brachman, R. (1983). What IS-A is and isn't: An analysis of taxonomic links in semantic networks, Computer 16(10): $30-36$.

Chaffin, R. and Herrmann, D.J. (1988). The nature of semantic relations: A comparison of two approaches, in M. Evens (Ed.), Relational Models of the Lexicon: Representing Knowledge in Semantic Networks, Cambridge University Press, New York, NY, pp. 289-334.

Deneubourg, J., Goss, S., Franks, N., Sendova-Franks, A., Detrain, C. and Chrétien, L. (1991). The dynamics of collective sorting: Robot-like ants and ant-like robots, Proceedings of the First International Conference on Simulation of Adaptive Behaviour: From Animals to Animats 1, MIT Press, Cambridge, MA, pp. 356-365.

Fernández, M.C., Menasalvas, E., Marban, O., Peña, J.M. and Millán, S. (2001). Minimal decision rules based on the Apriori algorithm, International Journal of Applied Mathematics and Computer Science 11(3): 691-704.

Greco, S., Matarazzo, B. and Słowiński, R. (2001). Rough sets theory for multicriteria decision analysis, European Journal of Operational Research 129(1): 1-47.

Handl, J., Knowles, J. and Dorigo, M. (2006). Ant-based clustering and topographic mapping, Artificial Life 12(1): 35-62.

Ishizu, S., Gehrmann, A., Nagai, Y. and Inukai, Y. (2007). Rough ontology: Extension of ontologies by rough sets, in M.J. 
Smith and G. Salvendy (Eds.), Human Interface and the Management of Information: Methods, Techniques and Tools in Information Design, Lecture Notes in Computer Science, Vol. 4557, Springer-Verlag, Berlin/Heidelberg, pp. $456-462$.

Köhler, J., Philippi, S., Specht, M. and Rüegg, A. (2006). Ontology based text indexing and querying for the semantic web, Knowledge-Based Systems 19(8): 744-754.

Lumer, E. and Faieta, B. (1994). Diversity and adaptation in populations of clustering ants, Proceedings of the Third International Conference on Simulation of Adaptive Behaviour: From Animals to Animats 3, MIT Press, Cambridge, MA, pp. 501-508.

Midelfart, H. and Komorowski, J. (2002). A rough set framework for learning in a directed acyclic graph, in J.J. Alpigini, J.F. Peters, A. Skowron and N. Zhong (Eds.), Rough Sets and Current Trends in Computing, Lecture Notes in Computer Science, Vol. 2475, Springer-Verlag, Berlin/Heidelberg, pp. 144-155.

Milstead, J.L. (2001). Standards for relationships between subject indexing terms, in C.A. Bean and R. Green (Eds.), Relationships in the Organization of Knowledge, Kluwer Academic Publishers, Dordrecht, pp. 53-66.

Neches, R., Fikes, R., Finin, T., Gruber, T., Patil, R., Senator, T. and Swartout, W. (1991). Enabling technology for knowledge sharing, AI Magazine 12(3): 36-56.

Pancerz, K. (2012a). Dominance-based rough set approach for decision systems over ontological graphs, in M. Ganzha, L. Maciaszek and M. Paprzycki (Eds.), Proceedings of FedCSIS'2012, Wroctaw, Poland, pp. 323-330.

Pancerz, K. (2012b). Toward information systems over ontological graphs, in J. Yao, Y. Yang, R. Słowiński, S. Greco, H. Li, S. Mitra and L. Polkowski (Eds.), Rough Sets and Current Trends in Computing, Lecture Notes in Artificial Intelligence, Vol. 7413, Springer-Verlag, Berlin/Heidelberg, pp. 243-248.

Pancerz, K. (2013a). Decision rules in simple decision systems over ontological graphs, in R. Burduk, K. Jackowski, M. Kurzyński, M. Woźniak and A. Zołnierek (Eds.), Proceedings of the 8th International Conference on Computer Recognition Systems CORES 2013, Advances in Intelligent Systems and Computing, Vol. 226, Springer International Publishing, Cham, pp. 111-120.

Pancerz, K. (2013b). Semantic relationships and approximations of sets: An ontological graph based approach, Proceedings of HSI'2013, Sopot, Poland, pp. 62-69.

Pancerz, K. (2014). Some remarks on complex information systems over ontological graphs, in A. Gruca, T. Czachórski and S. Kozielski (Eds.), Man-Machine Interactions 3, Advances in Intelligent Systems and Computing, Vol. 242, Springer International Publishing, Cham, pp. 55-62.

Pawlak, Z. (1991). Rough Sets: Theoretical Aspects of Reasoning about Data, Kluwer Academic Publishers, Dordrecht.
Roy, B. (1985). Méthodologie Multicritère d'Aide à la Décision, Economica, Paris.

Skowron, A. and Rauszer, C.M. (1992). The discernibility matrices and functions in information systems, in R.W. Slowinski (Ed.), Intelligent Decision Support: Handbook of Applications and Advances of the Rough Sets Theory, Kluwer Academic Publishers, Dordrecht, pp. 331-362.

Slowinski, R. and Vanderpooten, D. (1996). A generalized definition of rough approximations, IEEE Transactions on Knowledge and Data Engineering 12(2): 331-336.

Storey, V.C. (1993). Understanding semantic relationships, The VLDB Journal 2(4): 455-488.

Tadeusiewicz, R. (2010). Place and role of intelligent systems in computer science, Computer Methods in Materials Science 10(4): 193-206.

Tadeusiewicz, R. (2011). Introduction to intelligent systems, in B. Wilamowski and J. Irvin (Eds.), The Industrial Electronics Handbook: Intelligent Systems, CRC Press, Boca Raton, FL, pp. 1-1-1-12.

Wikisaurus (2013). The homepage, http://en.wiktionary.org/wiki/ Wiktionary:Wikisaurus

Winston, M. E., Chaffin, R. and Herrmann, D. (1987). A taxonomy of part-whole relations, Cognitive Science 11(4): 417-444.

Zadeh, L. (1996). Fuzzy logic = computing with words, IEEE Transactions on Fuzzy Systems 4(2): 103-111.

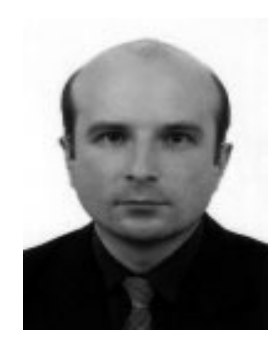

Krzysztof Pancerz, Rector of the University of Management and Administration in Zamość, Poland. He received his M.Sc. in 1998 from the Rzeszów University of Technology in electrical engineering and his Ph.D. in 2006 from the Institute of Computer Science, Polish Academy of Sciences, in computer science. His research interests concern computational intelligence, knowledge discovery, data mining, data semantics, and computer-aided diagnosis. He has published over 50 research papers in international journals, monographs and conference proceedings. He is a member of the International Rough Set Society.

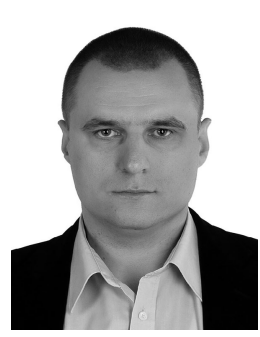

Arkadiusz Lewicki, Ph.D., assistant professor, lecturer and deputy head of the Chair of Information Systems Applications in the Department of Applied Informatics at the University of Information Technology and Management in Rzeszów. His research focuses mainly on swarm intelligence, neural networks and evolutionary computation. His current research interests also include metaheuristics and local search methods for combinatorial optimization and parallel and distributed computing. He is particularly interested in the application of statistical methods, design of experiments, and machine learning techniques. 


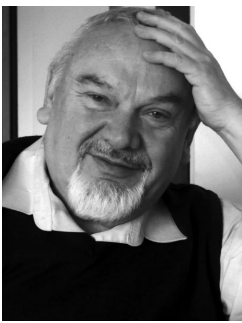

Ryszard Tadeusiewicz studied at the Electrical Engineering Department of the University of Mining and Metallurgy in Kraków (Poland), from which he graduated with honors (1971). Additionally, after receiving his degree in automatic control engineering, he studied at the Faculty of Medicine at the Medical Academy in Kraków, and undertook studies in the field of mathematical and computer methods in economics. He has written and published over 1000 scientific papers in prestigious Polish and foreign scientific journals as well as numerous conference presentations, both national and international. Professor Tadeusiewicz has also authored over 100 scientific monographs and books, including highly popular, frequently reprinted textbooks. He has been the supervisor of 76 doctoral theses and a reviewer of more than 300 doctoral dissertations.

Received: 22 March 2014

Revised: 8 August 2014

Re-revised: 28 January 2015 